\title{
CATALOGAÇÃO E TECNOLOGIA: INTERSEÇÕES COM A WEB SEMÂNTICA
}

\section{CATALOGUING AND TECHNOLOGY: INTERSECTIONS WITH THE SEMANTIC WEB}

\author{
Felipe Augusto Arakaki ${ }^{1}$ \\ Ana Carolina Simionato ${ }^{2}$ \\ Plácida Leopoldina Ventura Amorim da Costa Santos ${ }^{3}$
}

\begin{abstract}
RESUMO
Introdução: Os ambientes digitais se constituem em espaços importantes para geração e recuperação de recursos informacionais. Nesse contexto, é importante que o bibliotecário conheça as tecnologias disponíveis para melhorias nos processos de descrição, importação, transmissão, organização, indexação, armazenamento, localização, recuperação, visualização e preservação dos recursos informacionais. Objetivo: Nesse contexto, o objetivo deste trabalho é apresentar a inserção da tecnologia e suas projeções para Catalogação. Metodologia: $O$ método utilizado consiste em uma análise exploratória da literatura disponível sobre o tema proposto, permitindo a construção de um conhecimento teórico sobre a Catalogação frente as tecnologias, auxiliando na compreensão do contexto apresentado. Resultados: Como resultados é apresentado um panorama das mudanças do processo da Catalogação influenciado principalmente da proposta da família dos Requisitos Funcionais (FR) e da Web Semântica, em especial do Linked Data. Conclusões: Considera-se que o impacto da tecnologia sobre as atividades do bibliotecário, proporciona maior agilidade no processo de alimentação do catálogo.
\end{abstract}

Descritores: Catalogação. Tecnologia. Web Semântica.

\footnotetext{
${ }^{1}$ Doutorando em Ciência da Informação pela Universidade Estadual Paulista "Júlio Mesquita Filho" - (UNESP), Campus de Marília, SP. E-mail: fe.arakaki@gmail.com

${ }^{2}$ Doutora em Ciência da Informação pela Universidade Estadual Paulista "Júlio de Mesquita Filho" - UNESP/Marília. Docente do Departamento de Ciência da Informação da Universidade Federal de São Carlos (UFSCar). E-mail: simionato.ac@gmail.com

${ }^{3}$ Doutora em Letras - Semiótica e Linguística Geral pela Universidade de São Paulo (USP). Docente do Programa de Pós-Graduação em Ciência da Informação da Universidade Estadual Paulista (UNESP), Campus de Marília. E-mail: placidasantos@gmail.com
} 


\section{INTRODUÇÃO}

A configuração do ambiente digital apresenta uma gama de oportunidades aos profissionais que possuem competências do tratamento e representação da informação. Consequentemente, seu campo de atuação é expandido, pois, poderão explorar domínios específicos para organizar e representar um conjunto de recursos de acordo com as necessidades informacionais de indivíduos específicos.

Nesse contexto, a inserção das tecnologias no campo da informação significa além de um grande valor intrínseco, uma repercussão favorável, não só naqueles que trabalham diretamente com a área de informação, mas para todos aqueles que, no dia a dia, necessitam de informação. A dimensão tecnológica assumiu um papel preponderante nos processos sociais e econômicos, afetando inclusive a atuação profissional. Para o bibliotecário é imprescindível que desempenhe um papel ativo direcionado à orientação de seus usuários. Entre as suas funções, estão relacionadas a seleção das fontes de informação, acesso, uso e reuso informacional.

No ambiente multidisciplinar em que hoje se desenvolvem as tarefas de informação, o catalogador tem a vantagem intelectual de ser o conhecedor da essência dos processos de análise e tratamento do conhecimento registrado. Mas tem, entretanto, de estar apto para apropriar-se das ferramentas tecnológicas disponíveis para aperfeiçoamento e agilização de processos decisivos para o estabelecimento da organização e do tratamento da informação (SANTOS, 2010, p. 189).

Devido as características já apontadas, a Catalogação está alinhada as tecnologias de informação, no que diz a respeito do uso e reuso de registros bibliográficos para a construção de representações que possibilitem aos usuários encontrar, identificar, selecionar, adquirir ou obter e navegar no catálogo. Para isso, as tecnologias foram utilizadas para multidimensionar as possibilidades de acesso e recuperação da informação, como exemplo a interoperabilidade de dados bibliográficos. Dessa forma, o objetivo desse trabalho é apresentar a inserção da tecnologia e suas projeções para Catalogação. 
O método utilizado consiste em uma análise exploratória da literatura disponível sobre o tema proposto, permitindo a construção de um conhecimento teórico sobre a Catalogação frente as tecnologias, auxiliando na compreensão do contexto apresentado.

\title{
2 PANORAMA DA CATALOGAÇÃO E INFLUÊNCIA DAS TECNOLOGIAS
}

A Catalogação encontra nas tecnologias o suporte indispensável para criação de um canal de comunicação entre usuário e informação. Tal processo integra todas as atividades do ciclo de representação da informação que iniciase pelo planejamento da estrutura do sistema, atuando na infraestrutura e definição de metadados, até a busca e recuperação de informação via interface do sistema. De acordo com Boar (2002, p. 2)

\begin{abstract}
A tecnologia da informação é a preparação, coleta, transporte, recuperação, armazenamento, acesso, apresentação e transformação de informações em todas as suas formas (voz, gráficos, texto, vídeo e imagem). A movimentação de informação pode ocorrer entre seres humanos, entre humanos e máquina e/ou entre máquinas. $O$ gerenciamento da informação garante seleção, distribuição, administração, operação, manutenção e evolução dos bens de tecnologia da informação de forma coerente com as metas e objetivos da organização.
\end{abstract}

Os ambientes digitais constituem de espaços importantes para geração e recuperação de recursos informacionais. Nesse contexto, é extremamente importante para o catalogador conhecer as tecnologias disponíveis, os recursos digitais e os processos na ambiência digital para realizar: a descrição, a importação, a transmissão, a organização, a indexação, o armazenamento, a proteção e a segurança, a localização, a recuperação, a visualização, a impressão e a preservação dos recursos informacionais.

A padronização da descrição auxilia o catalogador na construção de representações mais completas e interoperáveis. Ressalta-se que ao construir estruturas eficientes e uma representação adequada é um modo de tornar os recursos informacionais disponíveis, acessíveis, recuperáveis e únicos. 
A construção de formas de representação atua como chave para o acesso aos recursos informacionais nos ambientes informacionais. Tais representações devem considerar que o recurso informacional pode estar fisicamente em outro espaço, como também, no próprio meio que the proporciona materialidade, o ambiente digital em que estão anexados à própria descrição. (ALVARENGA, 2001).

Contudo, o processo, os esquemas, as estruturas e os modelos de tratamento descritivo da informação (TDI) não são neutros (SANTOS, 2010), uma vez que, trazem no seu interior a visão de seus idealizadores e refletem posições ideológicas e políticas. A partir disso, o catalogador enfrenta dificuldades na implantação dos códigos e formatos de Catalogação e no efetivo acompanhamento do controle de qualidade dos registros bibliográficos produzidos. Outros problemas enfrentados estão relacionados a disponibilidade de recursos financeiros e humanos, problemas estruturais, divergências linguísticas, e do próprio objetivo durante o processo de construção da representação.

O bibliotecário, por meio da Catalogação, necessita dominar essas práticas para auxiliar a construção da representação para descrer detalhadamente o recurso informacional, adequando-se ao seu público, suporte e ambiente. Sendo assim, segundo Santos (2010, p. 193), a Catalogação consiste na

[...] disciplina e prática profissional, ela tem como missão construir formas de representação para alimentação de catálogos a partir da descrição padronizada de recursos informacionais, contemplando sua forma, seu conteúdo e o seu arranjo em acervos, de modo a tornar a unidade informacional única e multiplicar os pontos de acesso para a sua identificação, localização e recuperação; faz uso das tecnologias disponíveis nos mais diversos momentos históricos, na tentativa de descobrir caminhos para o aperfeiçoamento e otimização do trabalho cooperante e colaborativo.

Segundo Mey (1995) a Catalogação tem como objetivo tornar o recurso informacional único, por meio de uma estrutura sucinta e padronizada dos dados e informações de um recurso informacional. 
As novidades tecnológicas, principalmente as relacionadas com a informática motivaram algumas bibliotecas tradicionais especialmente nos países econômica e tecnologicamente mais desenvolvidos, ao aperfeiçoamento no uso das tecnologias de computação para o processamento eletrônico de dados, oferecendo acesso às bases de dados referenciais online, e, nos anos de 1960 a 1980, os On-line Public Access Catalogs (OPACs) passaram a ter uma maior presença no contexto das bibliotecas. (CASTRO; SANTOS, 2002, p. 63).

Alves e Santos (2013) apresentaram uma síntese da evolução da Catalogação, desde a Antiguidade, passando pela Idade Média até a Idade Contemporânea, relacionando as contribuições de cada período para construção da teoria da Catalogação, o desenvolvimento de padrões e o uso estratégico das tecnologias, conforme apontado no quadro 1.

Quadro 1 - Paralelo entre os períodos históricos da humanidade e os períodos históricos da Catalogação

\begin{tabular}{|c|c|}
\hline Períodos históricos da humanidade & Períodos históricos da Catalogação \\
\hline Antiguidade & Período Pré-tipográfico \\
\hline Idade Média & Período Pré-tipográfico \\
\hline Idade Moderna & Período Tipográfico \\
\hline \multirow{2}{*}{ Idade Contemporânea } & Período Semimecanizado \\
\cline { 2 - 2 } & Período Mecanizado \\
\cline { 2 - 2 } & Período Automatizado \\
\cline { 2 - 2 } & Período Tecnologias de rede e Web \\
\cline { 2 - 2 }
\end{tabular}

Fonte: Alves e Santos (2013, p. 25).

Na Idade Contemporânea, a Catalogação teve grandes avanços a partir da expansão da tecnologia, principalmente na década de 60 , com 0 desenvolvimento dos recursos computacionais. Nesse contexto, foi criado o formato de intercâmbio de dados, denominado Machine Readable Cataloging (MARC), que proporcionou o compartilhamento de registros bibliográficos legíveis por máquinas.

Entretanto, ao desenrolar de uma nova proposta de modelagem dos catálogos de bibliotecas no final da década de 90, nas décadas posteriores as estruturas dos instrumentos de representação como MARC21 e o código de 
Catalogação Anglo-American Cataloguing Rules (AACR2r) não estão mais compatíveis para esse novo cenário.

Ao longo da história da Catalogação, muitos debates, discussões foram realizadas para construção de esquemas de descrição bibliográficas e várias revisões desses esquemas vêm ocorrendo, como é o caso da proposta de um Código Internacional de Catalogação e da Resource Description and Access (RDA). A RDA foi desenvolvida pelo Joint Steering Committee for Development of RDA (JSC RDA) 4 com o objetivo de substituir o AACR2r.

Referindo-se aos aspectos que permeiam a Catalogação e a descrição bibliográfica na atualidade, o documento da Joint Steering Committee For Development of RDA (2005, p. 1, tradução nossa) explicam que:

As tecnologias digitais mudaram significativamente o ambiente em que bibliotecas, arquivos, museus e outras entidades que administram a informação, trabalham e mantêm suas bases de dados, as quais descrevem e proporcionam o acesso a recursos que integram seus acervos.

A proposta da RDA é fornecer uma estrutura flexível para descrever recursos informacionais - analógicos e digitais; estruturas emergentes das bases de dados e suportar tarefas dos catálogos propostas aos usuários. Isto é, a organização da RDA é baseada nos elementos descritivos derivados dos atributos associados com as entidades Functional Requirements for Bibliographic Records (FRBR) com o seguinte sentido: a obra é uma entidade abstrata, uma criação intelectual ou artística distinta; a expressão é a realização intelectual ou artística específica que assume uma obra ao ser realizada, excluindo-se aspectos de alteração da forma física; a manifestação é a materialização da expressão de uma obra, ou seja, seu suporte físico; e o item um único exemplar de uma manifestação. (CORRÊA; SANTOS, 2009).

Além do FRBR, a RDA está pautada na

[...] Declaração dos Princípios Internacionais de Catalogação, que oferece diretrizes para o desenvolvimento de códigos de Catalogação; os modelos conceituais Functional Requirements for Bibliographic Records (FRBR) (Requisitos Funcionais para

\footnotetext{
${ }^{4}$ O JSC RDA é composto por diversas instituições: The American Library Association (ALA), The Australian Committee on Cataloguing, The British Library, The Canadian Committee on Cataloguing, CILIP: Chartered Institute of Library and Information Professionals, Deutsche Nationalbibliothek e The Library of Congress, no intuito de manter e gerenciar a RDA.
} 
Registros Bibliográficos) e Functional Requirements for Authority Data (FRAD) (Requisitos Funcionais para Dados de Autoridade); e o esquema de visualização International Standard for Bibliographic Description (ISBD) (Padrão Internacional para Descrição Bibliográfica). (ASSUMPÇÃO; SANTOS, 2013, p. 205).

Com o uso da RDA em catálogos, a forma de criar e utilizar os dados bibliográficos será significativamente alterada. Oliver (2011) enfatiza que com a criação da RDA, o catálogo passa a não ser um depósito fechado, isto é, o catálogo contém "[...] muitos dados úteis, mas que estão aprisionados numa estrutura de registros específica da biblioteca [...]" (OLIVER, 2011, p. 121), e continua, "Quando os dados RDA são codificados em registros MARC num catálogo de biblioteca, esses dados não são visíveis e utilizáveis a não ser a partir do próximo catálogo." (OLIVER, 2011, p. 121).

O registro bibliográfico que era monolítico, como exemplo a ficha catalográfica orientada pelo AACR2r, passa a ser monobloco, composto por entidade e atributos. Isto é, uma descrição em um único registro como conhecemos, para uma estrutura que possibilita a descrição em partes, ou seja, permitindo um novo paradigma de ligação de acervos no ambiente digital (MARCONDES, 2016).

Nesse sentido, é criado atualizações para as orientações aos sistemas informacionais, ampliando o escopo do conceito da família FR, como o FRBR Library Reference Model (FRBR-LRM) para uma abrangência dos dados bibliográficos, como entendido em um sentido amplo e geral.

O modelo FRBR-LRM define uma única entidade de nível superior, nomeada como a 'coisa', uma generalização da entidade do [Functional Requirements for Subject Authority Data] FRSAD, além das necessidades da relação de assunto. Entre as entidades constituem de obra, expressão, manifestação, item, agente, nome, lugar, evento, pessoa, entidade coletiva, família e organização, esta última dividida em tipos de grupos e identificador do tipo de nome. (SIMIONATO, 2017, p. 219).

As modificações a serem realizadas nos catálogos existentes para as necessidades estruturais são grandes, principalmente para a restruturação sintática e semântica da composição dos registros e dados bibliográficos. Os dados bibliográficos deverão ser persistidos, para que tenham todo o potencial 
de utilização e aproveitamento nos relacionamentos. Consequentemente, a infraestrutura de armazenamento desses dados deverá ser bem planejada desde a sua concepção e manutenção durante todo o ciclo de gerenciamento de catálogos, para que todos os relacionamentos entre os dados sejam possíveis.

As iniciativas para catálogos direcionam a uma modelagem conceitual, todavia, há outros caminhos para a evolução dos catálogos como a inserção de ferramentas já discutidas pelos estudos da Web Semântica. Autores como Santos e Alves (2009), Baker et al. (2011); Marcondes (2012; 2016); Baker (2012); Catarino; Souza (2012); Méndez e Greenberg (2012); Silva, R. (2013), van Hooland; Verborgh (2014); Pomerantz (2015) e Arakaki (2016), entre outros, vêm discutindo a aplicação das tecnologias da Web Semântica no contexto do domínio bibliográfico.

Méndez e Greenberg (2012) apresentam uma evolução da Web Semântica, desde o controle de conteúdo da Internet em 1996. Para as autoras, outras ferramentas como Resource Description Framework (RDF) e RDF Schema foram surgindo, assim como o estabelecimento de padrões de metadados e de vocabulários para Web Semântica, como a estruturação do Dublin Core.

Assim, diversas linguagens computacionais foram desenvolvidas, a exemplo do Protocol and RDF Query Language (SPARQL) que proporciona consultas semânticas em conjuntos de dados. Além disso, o Simple Knowledge Organization System (SKOS) que se caracteriza por ser "[...] um modelo para expressar a estrutura básica e o conteúdo de sistemas de organização do conhecimento. SKOS é, como o nome representa, um sistema simples para a organização do conhecimento." (CATARINO, 2014). Para adequar ainda às necessidades estruturais da Web Semântica, tem a Web Ontology Language (OWL), que consiste em uma linguagem para definir e instanciar Ontologias na Web.

Segundo Arakaki (2016, p. 39) "Com o desenvolvimento das tecnologias, diversas foram as modificações da estrutura da Web Semântica e suas ferramentas expostas por Berners-Lee, Hendler e Lassila (2001)." Dessa forma, 
o diagrama das camadas da Web Semântica, conhecido como 'bolo de noiva' foi atualizado por Nowack (2009), que apresentou uma nova forma de estrutura em forma de cubo, colocando de um lado os conceitos e as abstrações e do outro lado as especificações e soluções tecnológicas desenvolvidas, como visualizado na figura 1.

Figura 1 - Tecnologias da Web Semântica

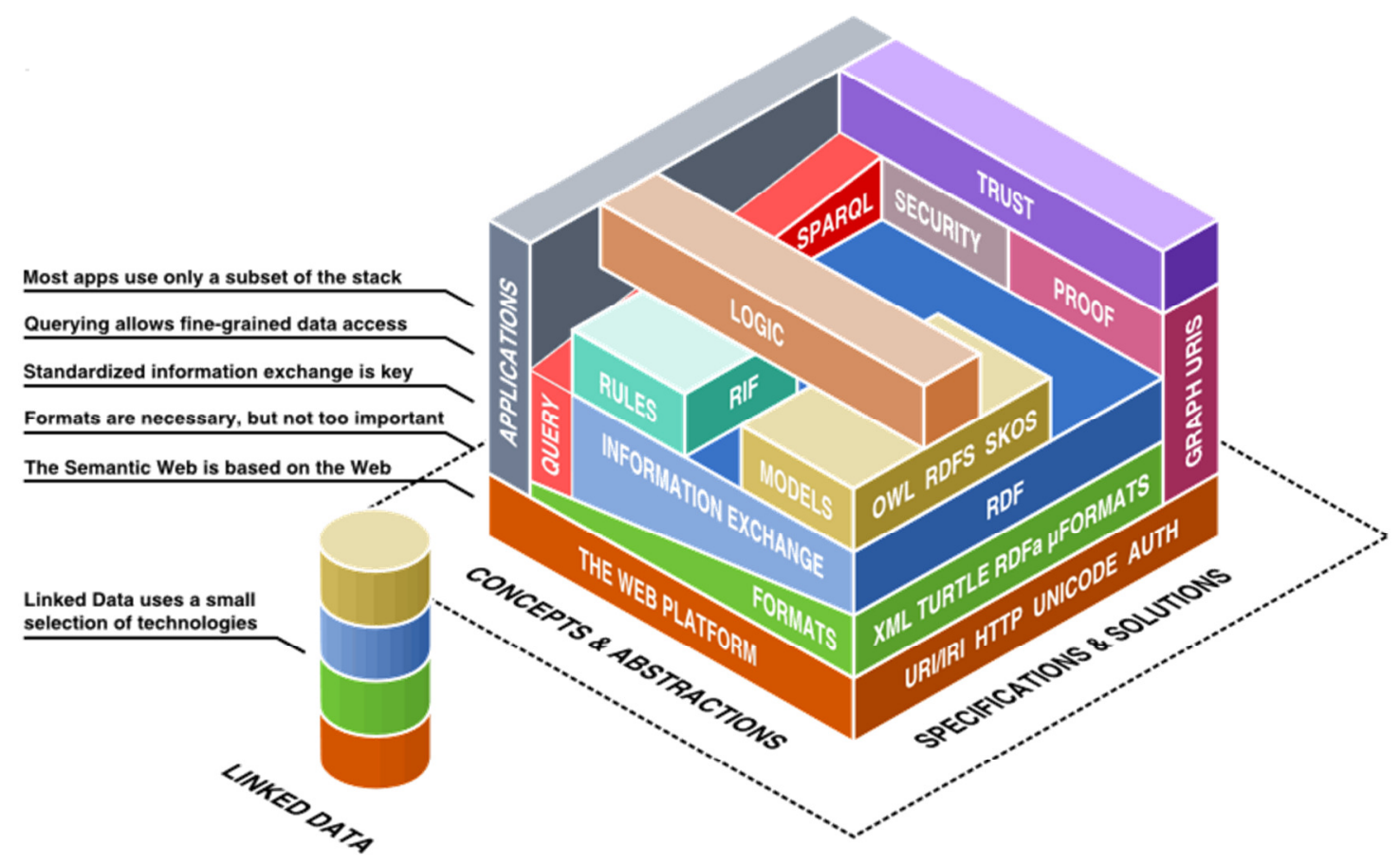

Fonte: Nowack (2009).

A figura 1 apresenta os pressupostos já conhecidos na representação de camadas, que incluem a camada sintática, camada XML (eXtensible Markup Language) e XML Schema, camada RDF e RDF Schema, camada de ontologias, camada lógica, e por fim, camada de prova. No entanto, Nowack (2009) destaca as tecnologias criadas até então para a base conceitual e apontadas como soluções para a extensão da Web, a plataforma da Web Semântica. No caso, a The Web Platform (A Plataforma Web) tem como solução URNRI HTTP e UNICODE AUTH, uma vez que a Web Semântica é baseada na Web. Para questões relacionadas aos formatos (formats) criaram a $\mathrm{XML}$, o Turtle, e o RDFa. A troca de informações (information exachange) é considerada pelo autor (NOWACK, 2009) como a chave do processo e é estabelecida pelo RDF. 
A composição das outras tecnologias (principalmente da troca de informações em RDF) permite que a busca (query) acesse os dados por meio do SPARQL. Os modelos estão relacionados à Web Ontology Language (OWL), Resource Description Framework Schema (RDFS), Simple Knowledge Organization System (SKOS), as Regras (rules) por meio do Rule Interchange Format (RIF) que são base para a camada de lógica. A segurança (security) e a prova (proof) estão relacionadas aos GRAPH URIS e são base para a estruturação da confiança (trust). (NOWACK, 2009).

Outro ponto destacado pela figura 1 é o Linked Data, que são princípios sobre

[...] como usar a Web para criar ligações entre os dados digitados a partir de diferentes fontes. [...] Tecnicamente, Linked Data refere-se a dados publicados na Web, de tal forma que é legível por máquina, o seu significado é explicitamente definido, ele está ligado a outros conjuntos de dados externos, e pode por sua vez ser ligados a partir de conjuntos de dados externos. (BIZER; HEATH; BERNERS-LEE, 2009, não paginado, tradução nossa).

Para que os dados possam ter uma qualidade e uma melhor estruturação, Berners-Lee (2006) definiu quatro princípios que são: 1) Use URIs como nomes para as coisas; 2) Use HTTP URIs para que as pessoas possam procurar esses nomes; 3) Quando alguém procurar um URI, forneça informações úteis, usando os padrões RDF e SPARQL; 4) Inclua links para outros URIs, para que eles possam descobrir mais coisas.

Para melhor viabilizar que esses dados sejam recuperados $e$ reutilizáveis no contexto dos Dados Abertos, Berners-Lee (2006) utilizou um sistema que classifica em até cinco estrelas a disponibilização de Linked Open Data, representado pela figura 2. 
Figura 2 - Cinco estrelas do Linked Open Data

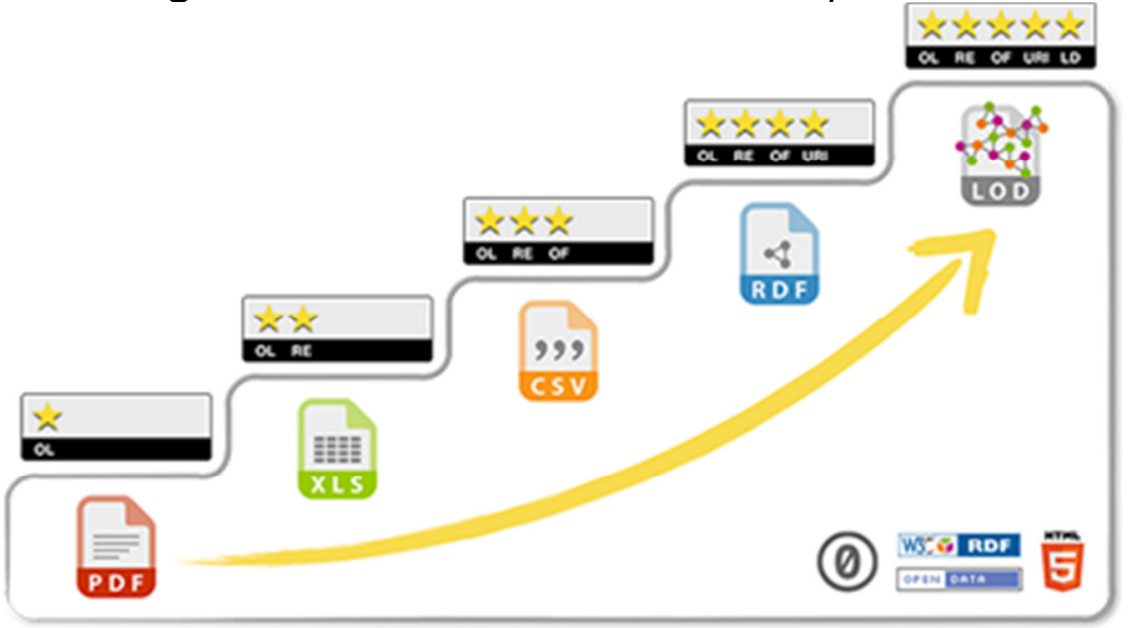

Fonte: Hausenblas (2012, não paginado).

O nível básico do Linked Open Data, ou seja, a primeira estrela deve-se disponibilizar os dados em qual quer formato na Web. Para conseguir duas estrelas, deve-se deixar os dados estruturados e que sejam legíveis por máquina. Se deixar disponível como dados estruturados legíveis por máquina em formato não proprietário, atinge três estrelas. Na quarta estrela, é necessário utilizar padrões abertos da World Wide Web Consortium (W3C) como RDF e SPARQL para identificar as coisas, de modo que as pessoas possam relacionar seu material. Por fim, para ter as cinco estrelas, é necessário, além das outras estrelas, relacionar os dados com outros dados de outros sites para assim, fornecer o contexto aos seus dados. (BERNERS-LEE, 2006).

Observa-se que a partir dessas novas tecnologias, foi criado um grupo de trabalho na W3C para discutirem as possibilidades de aplicação do Linked Data em bibliotecas denominado W3C Library Linked Data Incubator Group.

O objetivo do grupo foi de verificar as possibilidades de ampliação da interoperabilidade de dados de bibliotecas na Web. (BAKER et al., 2011). As principais recomendações do relatório do grupo foram: que os líderes de bibliotecas identifiquem conjuntos de dados como possíveis candidatos para expor dados ligados e fomentar uma discussão sobre dados abertos e seus direitos; que os organismos de normalização da biblioteca aumentem sua participação na padronização da Web Semântica; desenvolver padrões de dados de biblioteca que sejam compatíveis com o Linked Data; e disseminar 
padrões de projeto de melhores práticas adaptados ao Library Linked Data; que dados e projetistas de sistemas sejam mais bem utilizados com base na capacidade Linked Data; criar URIs para os itens em conjuntos de dados de biblioteca; desenvolver políticas para o gerenciamento de vocabulários RDF e suas URIs; e que bibliotecas expressem dados reutilizando ou mapeando vocabulários existentes em Linked Data; que bibliotecários e arquivistas preservem conjuntos de elementos de dados e vocabulários de valores e aplicar a experiência em curadoria de bibliotecas e preservação em longo prazo os conjuntos de dados Linked Data (BAKER et al., 2011).

Essas recomendações reforçam a necessidade dos profissionais da informação, principalmente aqueles que lidam diretamente com a Catalogação, descrição e os metadados, estarem se aperfeiçoando e utilizando as tecnologias disponíveis para que possibilitem melhorias aos catálogos e a localização, acesso, uso e reuso de recursos informacionais.

\section{REFLEXÕES FINAIS}

Em síntese, o processo de Catalogação é um dos responsáveis por unir e colocar à disposição recursos informacionais a todos os usuários. Para que isso aconteça adequadamente é necessário que o profissional execute uma representação mínima do acervo de sua responsabilidade. Entretanto, com as novas possibilidades de reuso dos dados bibliográficos, ressalta-se o impacto da tecnologia sobre as atividades do bibliotecário, proporcionando maior agilidade no processo de alimentação do catálogo.

As mudanças não serão imediatas, mas é importante que elas sejam despertadas. Alguns softwares comerciais para as bibliotecas já estão se preparando para suportar registros e relacionamentos em RDF e RDA. Nesse sentido, irão ampliar as possibilidades de uso e reuso de dados bibliográficos de diferentes sistemas.

Por fim, é importante destacar que o profissional deve buscar um aperfeiçoamento constante, seja em cursos extras ou mesmo pela rotina de contextualização dos desenvolvimentos de sua área. A formação da graduação 
não promoverá a resolução de todas as incógnitas e novos preceitos da área, por essa razão, o profissional deve buscar incessantemente a atualização de seus currículos. No entanto, a demanda de novas temáticas é inversa a burocracia de alteração dos projetos pedagógicos ou mesmo da carga horária dos bacharelados em Biblioteconomia e Ciência da Informação.

\section{REFERÊNCIAS}

ALVARENGA, L. A teoria do conceito revisitada em conexão com ontologias e metadados no contexto das bibliotecas tradicionais e digitais. Datagramazero - Revista de Ciência da Informação, v.2, n. 6, dez. 2001. Disponível em: <http://basessibi.c3sl.ufpr.br/brapci/index.php/article/view/0000001252/f08a9a9 553736ff081222d0af5631e20>. Acesso em: 15 set. 2017.

\section{ALVES, R. C. V.; SANTOS, P. L. V. A. C. Metadados no domínio}

bibliográfico. Rio de Janeiro: Intertexto, 2013.

ARAKAKI, F. A. Linked Data: ligação de dados bibliográficos. 2016. $144 f$. Dissertação (Mestrado em Ciência da Informação) - Universidade Estadual Paulista Júlio de Mesquita Filho, Faculdade de Filosofia e Ciências, Marília, 2016. Disponível em: <https://repositorio.unesp.br/handle/11449/147979>. Acesso em: 15 set. 2017.

ASSUMPÇÃO, F. S.; SANTOS, P. L. V. A. C. A utilização do Resource Description and Access (RDA) na criação de registros de autoridade para pessoas, famílias e entidades coletivas. Encontros Bibli: Revista Eletrônica de Biblioteconomia e Ciência da Informação, Florianópolis, v. 18, n. 37, p. 203226, 2013. Disponível em:

<https://periodicos.ufsc.br/index.php/eb/article/view/26536>. Acesso em: 15 set. 2017.

BAKER, T. Libraries, languages of description, and linked data: a Dublin Core perspective. Library Hi Tech, v. 30, n. 1, p. 116-133, mar. 2012. Disponível em: <http://www.emeraldinsight.com/doi/abs/10.1108/07378831211213256/>. Acesso em: 15 set. 2017.

BAKER, T. et al. Library Linked Data Incubator Group Final Report. W3C Incubator Group Report, 2011. Disponível em: <http://www.w3.org/2005/Incubator/lld/XGR-lld-20111025/>. Acesso em: 15 set. 2017. 
BERNERS-LEE, T. Linked Data: Design Issues. [S.I.]: W3C, 2006. Disponível em: <http://www.w3.org/Designlssues/LinkedData.html>. Acesso em: 15 set. 2017.

BERNERS-LEE, T.; HENDLER, J.; LASSILA, O. The Semantic Web. Scientific American, p. 29-37, may. 2001.

BIZER, C.; HEATH, T.; BERNERS-LEE, T. Linked data: the story so far. International Journal on Semantic Web and Information Systems, v. 5, n. 3, p. 1-22, 2009. Disponível em: <http://tomheath.com/papers/bizer-heath-bernerslee-ijswis-linkeddata.pdf>. Acesso em: 15 set. 2017.

BOAR, B. Tecnologia da informação: a arte do planejamento estratégico. 2.ed. São Paulo: Berkeley, 2002.

CASTRO, F. F.; SANTOS, P. L. V. A. C. Conversão Retrospectiva de Registros Bibliográficos. In: FUJITA, M. S. L. (Org.). A dimensão social da biblioteca digital na organização e acesso ao conhecimento: projetos, produtos e relatos de experiências. São Paulo: Sistema Integrado de Bibliotecas da USP, Dep. Técnico IBICT, 2002, v. 2, p. 63-87.

CATARINO, M. E. Simple Knowledge Organization System: construindo sistemas de organização do conhecimento no contexto da Web Semântica. Informação \& Tecnologia (ITEC): Marília/João Pessoa, v.1, n.1, p. 17-28, jan./jun., 2014. Disponível em <http://periodicos.ufpb.br/ojs/index.php/itec/article/view/19307>. Acesso em: 15 set. 2017.

CATARINO, M. E.; SOUZA, T. B. de. A representação descritiva no contexto da web semântica. Transinformação, Campinas, v. 24, n. 2, p. 77-90, mai./ago. 2012. Disponível em: <http://periodicos.puccampinas.edu.br/seer/index.php/transinfo/article/view/766/746>. Acesso em: 15 set. 2017.

CORRÊA, R. M. R.; SANTOS, P. V. L. A. Catalogação: trajetória para um novo código internacional. Niterói: Intertexto, 2009. 80p.

HAUSENBLAS, M. Exploiting Linked Data to Build Web Applications. IEEE Internet Computing, v. 13, p. 68-73, jul./ago. 2009. Disponível em: $<$ http://doi.ieeecomputersociety.org/10.1109/MIC.2009.79 >. Acesso em: 15 set. 2017.

JOINT STEERING COMMITTEE FOR DEVELOPMENT OF RDA. RDA: Resource Description and Access. [S.I.]: JSC, 2007. 12 p. Disponível em: <http://www.rda-jsc.org/archivedsite/docs/5rda-prospectusrev4.pdf>. Acesso em: 03 dez. 2017. 
MARCONDES, C. H. "Linked data" - dados interligados - e interoperabilidade entre arquivos, bibliotecas e museus na web. Encontros Bibli: revista eletrônica de biblioteconomia e ciência da informação, v. 17, n. 34, p. 171-192. jun. 2012. Disponível em:

<https://periodicos.ufsc.br/index.php/eb/article/view/1518-

2924.2012v17n34p171>. Acesso em: 15 set. 2017.

MARCONDES, C. M. Interoperabilidade entre acervos digitais de arquivos, bibliotecas e museus: potencialidades das tecnologias de dados abertos interligados. Perspectivas em Ciência da Informação, v. 21, n. 2, p. 61-83, abr./jun. 2016. Disponível em:

$<$ http://portaldeperiodicos.eci.ufmg.br/index.php/pci/article/view/2735>. Acesso em: 15 set. 2017.

MENDEZ, E. GREENBERG, J. Linked data for open vocabularies and HIVE's global framework. El profesional de la información, v. 21, n. 3. mai./jun. 2012. Disponível em:

<http://recyt.fecyt.es/index.php/EPI/article/view/epi.2012.may.03/17916>. Acesso em: 7 abr. 2016.

MEY, E. S. A. Introdução à Catalogação. Brasília: Briquet de Lemos, 1995.

NOWACK, B. The Semantic Web Technology Stack (not a piece of cake...). Linked Data Developer. 2009. Disponível em:

$<$ http://linkeddatadeveloper.com/Projects/Linked-Data/media/fig11.2.png>. Acesso em: 7 abr. 2016.

OLIVER, C. Introdução à RDA: um guia básico. Brasília: Briquet de Lemos / Livros, 2011.

POMERANTZ, J. Metadata. USA: The MIT press essential knowledge series, 2015.

SANTOS, P. L. V. A. C. Catalogação e ética no ambiente colaborativo e de empoderamento das redes informacionais. In: FREIRE, G.H.A. Ética da informação: conceitos, abordagens, aplicações. João Pessoa: Ideia, 2010.

SANTOS, P. L. V. A. C.; ALVES, R. C. V. Metadados e Web Semântica para estruturação da Web 2.0 e Web 3.0. DataGramaZero, Rio de Janeiro, v. 10, n. 6. dez. 2009. Disponível em: <http://www.dgz.org.br/dez09/Art_04.htm>. Acesso em: 21 ago. 2017.

SILVA, R. E. As tecnologias da Web Semântica no domínio bibliográfico. 2013. 134 f. Dissertação (Mestrado em Ciência da Informação) - Universidade Estadual Paulista Júlio de Mesquita Filho, Faculdade de Filosofia e Ciências, Marília, 2013. Disponível em: <http://hdl.handle.net/11449/93653>. Acesso em: 21 ago. 2017. 
SIMIONATO, A. C. Família FR, BIBFRAME e Linked Data: integração de dados bibliográficos. In: ZAFALON, Z. R.; DAL'EVEDOVE, P. R. Perspectivas da representação documental: discussão e experiências. São Carlos: CPOI/UFSCar, 2017.

VAN HOOLAND, S.; VERBORGH, R. Linked data for libraries, archives and museums: how to clean, link and publish your metadata. UK: Facet Publishing, 2014.

\title{
CATALOGUING AND TECHNOLOGY: INTERSECTIONS WITH THE SEMANTIC WEB
}

\begin{abstract}
Introduction: Digital environments are in important spaces for the generation and recovery of informational resources. In this context, it is important that the librarian knows the technologies available for improvements in the processes of description, import, transmission, organization, indexing, storage, localization, recovery, visualization and preservation of informational resources. Objective: In this context, the objective of this work is to present the insertion of the technology and its projections for cataloguing. Methodology: The method used consists of an exploratory analysis of the literature available on the proposed theme, allowing the construction of a theoretical knowledge about the cataloguing of the technologies, helping in the understanding of the context presented. Results: As a result, an overview of the changes in the cataloging process is presented mainly influenced by the family proposal of Functional Requirements (FR) and the Semantic Web, especially Linked Data. Conclusions: It is considered the impact of technology on the activities of the librarian, provides greater agility in the process of feeding the catalog.
\end{abstract}

Descriptors: Cataloguing. Technology. Semantic Web.

\section{CATALOGACIÓN Y TECNOLOGÍA: INTERSECCIONES CON LA WEB SEMÁNTICA}

\section{RESUMEN}

Introducción: Los ambientes digitales se constituyen en espacios importantes para la generación y recuperación de recursos informacionales. En este contexto, es importante que el bibliotecario conozca las tecnologías disponibles para mejoras en los procesos de descripción, importación, transmisión, organización, indexación, almacenamiento, localización, recuperación, visualización y preservación de los recursos informacionales. Objetivo: En este contexto, el objetivo de este trabajo es presentar la inserción de la tecnología y sus proyecciones para Catalogación. Metodología: El método utilizado consiste en un análisis exploratoria de la literatura disponible sobre el tema propuesto, permitiendo la construcción de un conocimiento teórico sobre la Catalogación frente a las tecnologías, ayudando en la comprensión del contexto presentado. Resultados: Como resultados se presenta un panorama de los 
cambios en el proceso de Catalogación influido principalmente de la propuesta de la familia de los Requisitos Funcionales (FR) y de la Web Semántica, en especial de Linked Data. Conclusiones: Se considera que el impacto de la tecnología sobre las actividades del bibliotecario, proporciona mayor agilidad en el proceso de alimentación del catálogo.

Descriptores: Catalogación. Tecnología. Web Semántica.

Recebido: 20.10 .2017

Aceito: 28.12 .2017 